\title{
CIÊNCIA'NATURA
}

\section{Perfis verticais dos espectros das velocidades turbulentas em uma torre micrometeorológica de $140 \mathrm{~m}$}

\section{Vertical profiles of the turbulent velocity spectra for a $140 \mathrm{~m}$ micrometeorological tower}

${ }^{1}$ Otávio Costa Acevedo, ${ }^{1}$ Pablo Eli Soares de Oliveira, ${ }^{1}$ Claudio Alberto Teichrieb,

${ }^{1}$ Franciano Scremin Puhales, ${ }^{1}$ Luis Gustavo Nogueira Martins, ${ }^{1}$ Gervásio Annes Degrazia, ${ }^{1}$ Samuel Martins Silva e ${ }^{1}$ Rafael Maroneze

Universidade Federal de Santa Maria, Santa Maria, RS, Brasil

\section{Resumo}

Espectros de multirresolução das componentes horizontais e verticais do vento observados em uma torre micrometeorológica de $140 \mathrm{~m}$, localizada na cidade de Linhares, ES, a $4 \mathrm{~km}$ da costa e ao lado de uma usina termoelétrica, são analisados. No período noturno, o máximo de energia está localizado nas maiores escalas temporais analisadas, estando associado a processos não turbulentos de baixa frequência. Durante o dia, as escalas temporais associadas aos máximos espectrais das componentes horizontais do vento variam pouco com a altura acima de $20 \mathrm{~m}$. Para a componente vertical a escala destes máximos é diretamente relacionada com a altura da observação.

Palavras-chave: energia cinética turbulent, espectros de multirresolução, máximos espectrais.

Abstract

Multiresolution spectra of both horizontal and vertical wind components observed at a 140-m micrometeorological tower are analysed. The tower is located at the township of Linhares, ES, Brazil, at $4 \mathrm{~km}$ from the coast and nest to a thermal power plant. At nighttime, the spectral maximum is located at the largest temporal scales analyzed, being therefore related to nonturbulent low-frequency processes. During the day, spectral maxima of the horizontal wind components show little variation above $20 \mathrm{~m}$. For the vertical component, on the other hand, the temporal scale of such maxima increases steadily with height.

Keywords: multiresotution spectra, spectral máxima, turbulent kinetic energy. 


\section{Introdução}

Espectros de turbulência fornecem informações sobre a distribuição da energia cinética turbulenta em escalas temporais ou espaciais. São bastante úteis para uma grande quantidade de aplicações, incluindo o desenvolvimento de modelos de dispersão, nos quais as parametrizações de difusividades turbulentas são frequentemente feitas em termos das escalas associadas aos máximos espectrais (Degrazia et al., 2000). A análise espectral também pode auxiliar a compreensão de características do escoamento na camada limite planetária ao identificar as escalas em que o escoamento é ou não turbulento, e como as escalas variam em respeito a forçantes externos, como transições ou outros (Vickers e Mahrt, 2006; Acevedo et al., 2014). Os espectros das componentes da velocidade turbulenta na camada limite atmosférica são analisados desde que as medidas em campo se tornaram tecnicamente possíveis. Kaimal et al. (1972) mostrou, a partir de dados do experimento clássico de Kansas (Haugen et al., 1971) que em condições neutras e estáveis o espectro da componente vertical é função do parâmetro de estabilidade e que a frequência dos máximos espectrais é relacionada com a razão, onde é o vento médio e é a altura da observação. Em condições instáveis, porém, não é possível distinguir um padrão, sendo possível que outras escalas, como a espessura da camada limite, sejam relevantes. Para as componentes horizontais, padrões semelhantes foram observados por Kaimal et al. (1972) que, adicionalmente identificou a ocorrência de máximos de mais baixa frequência. No presente trabalho, espectros das componentes horizontais e verticais da velocidade turbulenta obtidos através da decomposição de multirresolução (Mallat, 1989; Howell e Mahrt, 1997) são determinados e analisados para 11 níveis de turbulência entre a superfície e $140 \mathrm{~m}$ de altura em uma torre micrometeorológica. A análise foca na determinação do pico espectral e o que determina as escalas temporais nas quais esse pico ocorre.

\section{Observações e análise}

Os dados utilizados provém da torre micrometeorológica de Linhares, localizada junto à Usina Termoelétrica da Linhares Geração SA, através de um projeto de Pesquisa \& Desenvolvimento. A torre está localizada $250 \mathrm{~m}$ ao norte do prédio principal da Usina, e a $4 \mathrm{~km}$ do litoral. Os dados de turbulência provém de 11 níveis verticais, nas alturas de 1, 2, 5, 9, 20, 37, 56, 76, 94, 113 e 132 m. Os instrumentos utilizados são anemômetros sônicos tridimensionais CSAT3B (Campbell Sci., Inc.), exceto em 20 m, onde são coletados por um IRGASON (Campbell Sci., Inc.) As medidas iniciaram em agosto de 2016, e nesse trabalho são utilizadas as feitas até o final de janeiro de 2017. Apenas as condições em que o vento tinha componente de norte para sul e, portanto, não era afetado pelos prédios da usina, foram utilizados. Um total de 57 dias em que os dados em todos os níveis estiveram com boa qualidade durante as 24 horas do período foram utilizados para a análise aqui apresentada.

Os espectros foram determinados para cada período de $1 \mathrm{~h}$, utilizando a decomposição em multirresolução (Mallat, 1989; Howell e Mahrt, 1997). Essa técnica permite melhor definição dos picos espectrais nas baixas frequências, que espectros de Fourier. Para cada série de $1 \mathrm{~h}$, os espectros poram interpolados por uma média móvel, da qual o pico foi identificado, utilizando um procedimento semelhante ao que é explicado por Campos et al. (2009). Neste trabalho, os espectros das duas componentes horizontais, e, são combinados em um único espectro horizontal.

\section{Resultados}

Os espectros médios do dia 26 de outubro de 2016 (figura 1) são representativos das características gerais observadas nos casos em que o vento médio teve componente meridional de norte. Os espectros das duas componentes horizontais combinadas apresentam um pico associado aos movimentos turbulentos e máximos de baixa frequência, associados a movimentos não turbulentos e que ocorrem nas máximas escalas temporais incluídas na presente análise (figura 1, painéis superiores). Para a componente vertical, por sua vez, apenas o pico turbulento é aparente (figura 1, painéis inferiores). A escala em que os máximos espectrais associados a processos turbulentos ocorrem depende da componente analisada, período do dia e altura. Em todos os casos, há uma diferença bem evidente entre as escalas dos máximos espectrais observadas até a altura de $9 \mathrm{~m}$ e de $20 \mathrm{~m}$ de altura para cima. Essa distinção é muito provavelmente associada a um conjunto de árvores de altura em torno de $10 \mathrm{~m}$ localizadas a nordeste da torre, que muito provavelmente fazem com que as observações mais baixas estejam ainda na subcamada rugosa. Para as componentes horizontais durante o dia (figura 1, painel superior esquerdo), o máximo espectral turbulento ocorreu em escalas temporais entre 1 e 10 s, enquanto em níveis mais elevados ocorreu em escalas próximas a $100 \mathrm{~s}$, sem variação significativa com a altura para esses níveis mais elevados. Durante a noite, estes mesmos espectros horizontais não apresentaram variação significativa da escala de seus máximos nas alturas até $9 \mathrm{~m}$, mas acima deste nível os máximos ocorreram em escalas temporais em torno de $10 \mathrm{~s}$, que é uma ordem de magnitude menor que as escalas observadas durante o dia. A componente vertical apresenta algumas diferenças importantes. Como no caso horizontal, as escalas dos máximos turbulentos são sempre menores entre 1 e $9 \mathrm{~m}$ que entre 9 e $132 \mathrm{~m}$, tanto no caso diurno (figura 1, painel inferior esquerdo) como no noturno (painel inferior direito). Porém, em contraste com o caso horizontal, o máximo espectral turbulento na vertical cresce continuamente com a altura, em concordância com resultados clássicos (Panofsky e Dutton, 1984), que associam este comportamento com a barreira mecânica que a superfície representa para vórtices na vertical, mas não na horizontal. 


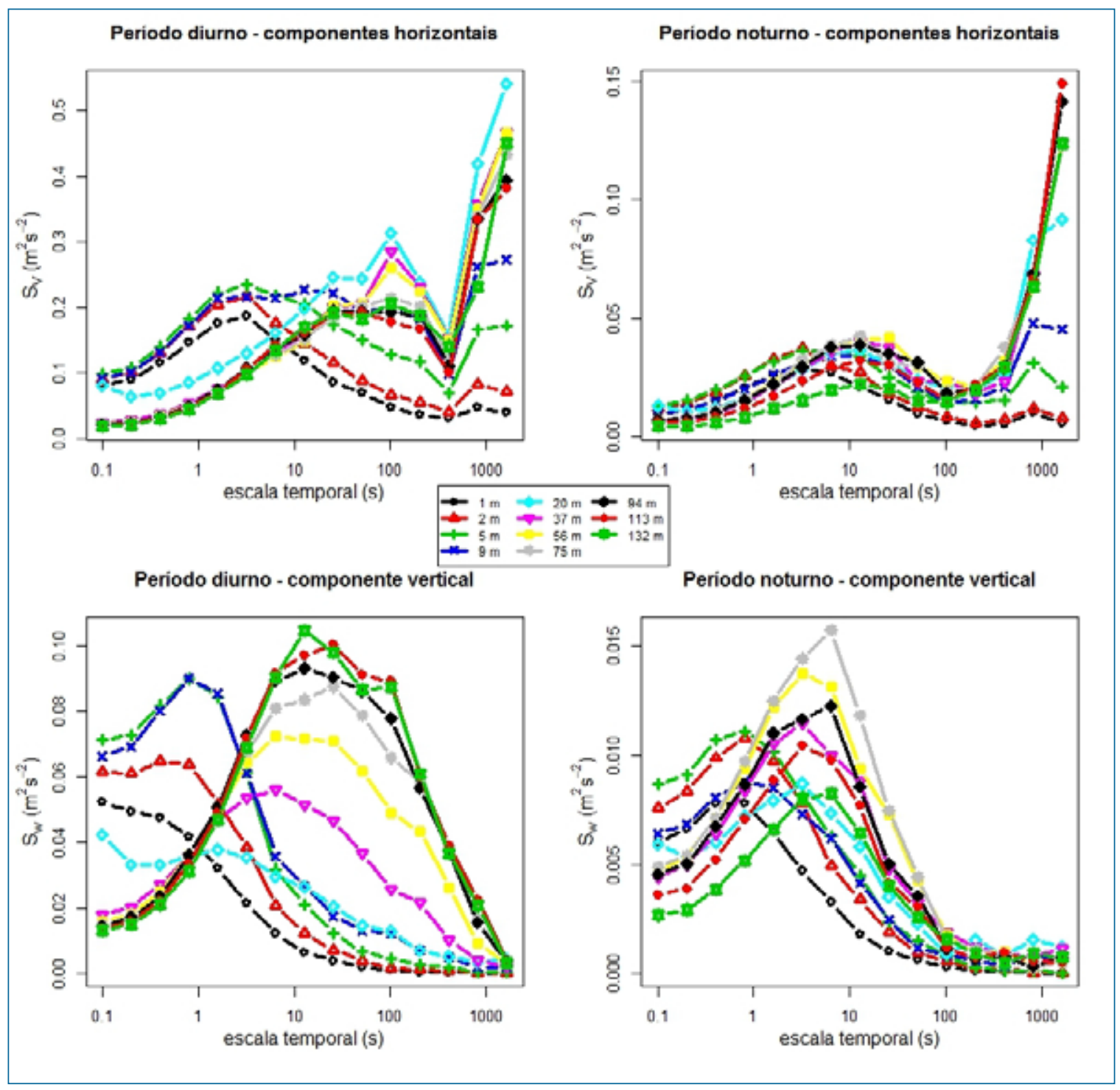

Figura 1: Espectros médios de multiresolução das componentes horizontais da velocidade turbulenta (painéis superiores) e da componente vertical (painéis inferiores) para períodos diurno (painéis da esquerda) e noturno (painéis da direita) do dia 26 de outubro de 2016, e para os diferentes níveis verticais, de acordo com a legenda.

Além da dependência com a vertical, em alguns casos as escalas temporais dos máximos espectrais apresentam uma dependência bem evidente da velocidade média do vento (figura 2). Essa dependência é bem estabelecida, e motivou inclusive que em trabalhos clássicos (Kaimal et al., 1972; Højstrup, 1981) os espectros fossem apresentados em termos de uma frequência adimensional, onde n é a frequência natural, z é a altura da observação e U é a velocidade do vento médio. De maneira geral, as escalas dos máximos espectrais diminuem com a velocidade do vento, mas essa diminuição pode ser mais ou menos marcante. A maior dependência com a velocidade do vento ocorre em níveis mais baixos no período noturno. Para as componentes horizontais as escalas dos máximos apresentam em todos os níveis acima de $20 \mathrm{~m}$ a mesma tendência de diminuição com a velocidade do vento, variando de cerca de $200 \mathrm{~s}$ para ventos de $5 \mathrm{~m} \mathrm{~s}-1$ para $50 \mathrm{~s}$ para ventos superiores a $10 \mathrm{~m} \mathrm{~s}^{-1}$ (figura 2 , painel superior esquerdo). Na componente vertical, uma dependência semelhante é observada em relação ao vento médio, mas fica evidente que em níveis mais elevados os máximos espectrais ocorrem em escalas temporais progressivamente maiores, para qualquer velocidade do vento (figura 2, painel inferior esquerdo).

A única condição em que a escala temporal do máximo espectral sempre apresenta uma relação linear com é para a componente vertical no período diurno (figura 3, painel inferior esquerdo). Neste caso, existem dois comportamentos distintos, mas nos dois a relação é clara. Para alturas até $20 \mathrm{~m}$, a escala do pico espectral vertical cresce pouco com e para níveis superiores este crescimento é acentuado, e independente da altura. No mesmo período diurno, a escala temporal do máximo espectral horizontal (figura 3 , painel superior esquerdo) depende linearmente de até $9 \mathrm{~m}$, mas acima deste nível a relação passa a ser dependente da altura, e não 


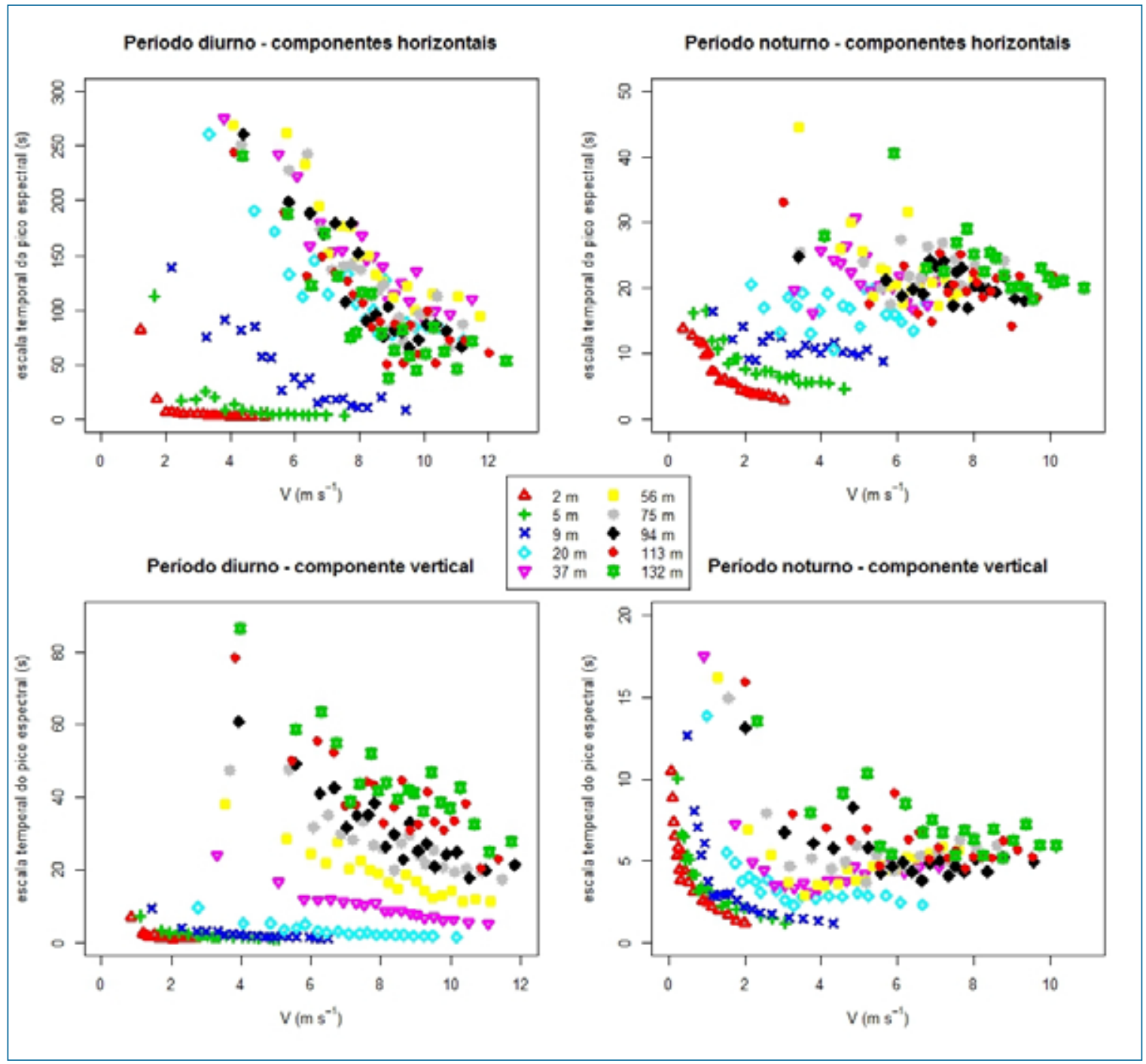

Figura 2: Escala temporal do máximo espectral de (painéis superiores) e (painéis inferiores) em função da velocidade média do vento para os períodos diurno (painéis da esquerda) e noturno (painéis da direita) e para os diferentes níveis verticais, de acordo com a legenda. Para cada nível, são apresentados 20 pontos que correspondem a médias de blocos dos valores observados, onde cada bloco contém igual número de pontos.

universal, estando esse comportamento associado à independência dessa escala na vertical em condições de mistura convectiva, conforme pode ser também visto na figura 2 (painel duperior direito). No período noturno, por sua vez, há aparentemente a indicação que a escala do pico horizontal segue a escala de até $37 \mathrm{~m}$, e é independente deste acima desta altura. Uma análise mais detalhada é necessária para esclarecer o que exerce o controle sobre esse comportamento, especialmente porque Acevedo et al. (2018) mostraram que há dois regimes distintos durante a noite: a primeira parte, que na média dura até 0200 HL é fracamente estável, se tornando muito estável a partir daí.

\section{Conclusões}

Os dados de turbulência da torre de Linhares constituem um conjunto muito rico de informações devido à sua cobertura vertical e temporal. Por esse motivo, permitem inferir características do escoamento turbulento com riqueza de detalhes melhor que em campanhas experimentais anteriores. O presente trabalho mostrou que as escalas temporais dos picos espectrais das componentes horizontal e vertical do vento turbulento dependem da altura e da velocidade do vento. Em algumas condições essa dependência é bem comportada, como no caso diurno. No caso noturno, essa relação é menos comportada e estudos mais detalhados são importantes para esclarecer o que exerce esse controle. Estes resultados tem um grande potencial de aplicabilidade em modelos de dispersão de escalares, que utilizam essas escalas em suas parametrizações. 


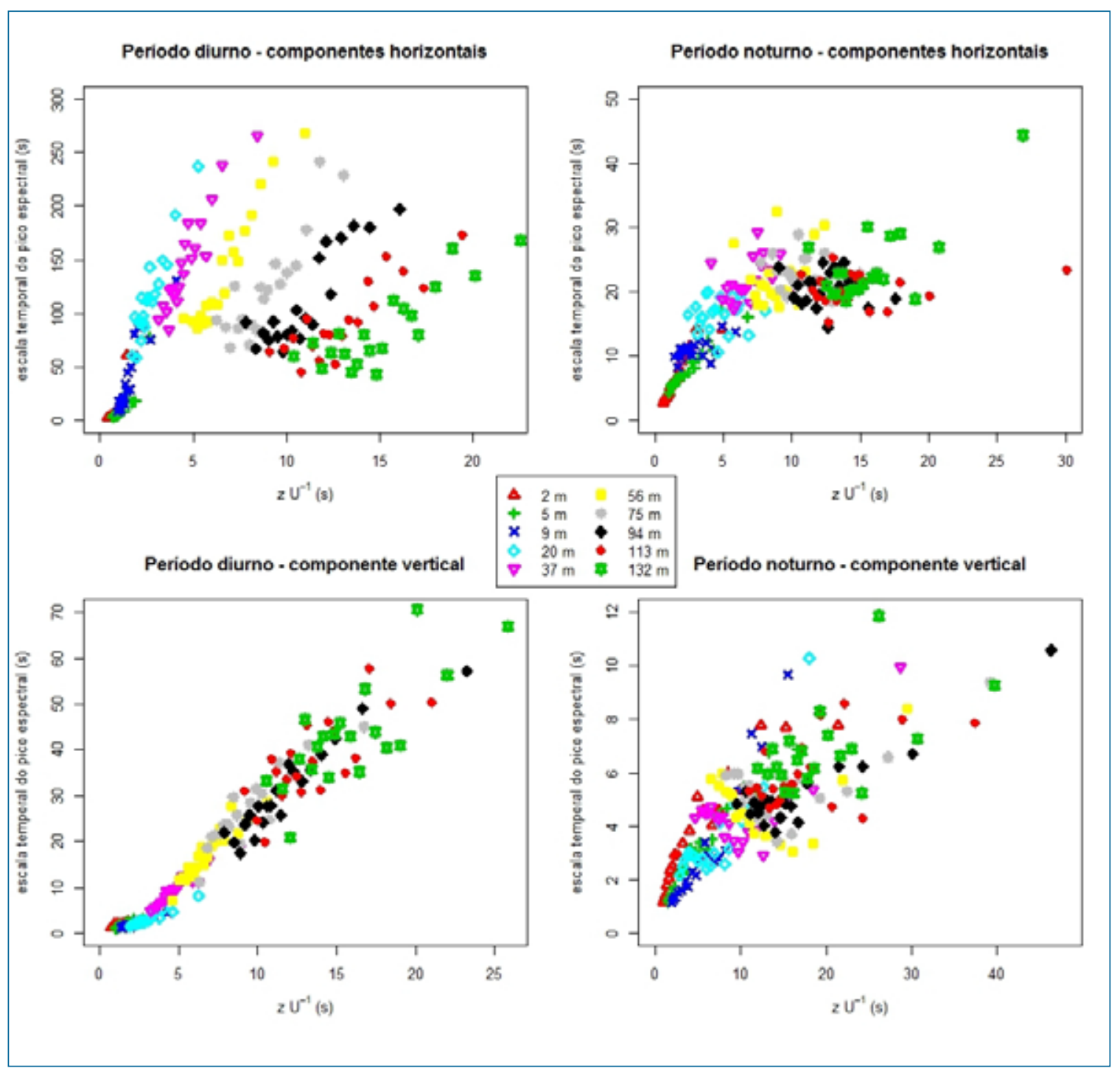

Figura 3: Relação entre a escala temporal média observada para o pico espectral e a escala para as componentes horizontal (painéis superiores) e vertical (painéis inferiores) do vento, nos períodos diurno (painéis da esquerda) e noturno (painéis da direita) nos diferentes níveis verticais, de acordo com a legenda. Para cada nível, são apresentados 20 pontos que correspondem a médias de blocos dos valores observados, onde cada bloco contém igual número de pontos.

\section{Agradecimentos}

Este estudo foi desenvolvido no contexto de um projeto de Pesquisa \& Desenvolvimento patrocinado pelas empresas Linhares Geração S. A. e Termoelétrica Viana S. A., denominado "Desenvolvimento de um modelo operacional para simulação em tempo real da dispersão atmosférica de poluentes emitidos por termelétrica a gás natural. O projeto se insere no programa de Pesquisa \& Desenvolvimento, regulado pela Agência Nacional de Energia Elétrica. Os autores agradecem o apoio proporcionado por essas empresas no desenvolvimento do trabalho.

\section{Referências}

Acevedo, O. C., Costa, F. D., Oliveira, P. E., Puhales, F. S., Degrazia, G. A., Roberti, D. R. (2014). The influence of submeso processes on stable boundary layer similarity relationships. Journal of the Atmospheric Sciences, 71(1), $207-225$.

Acevedo, O. C., Degrazia, G. A., Puhales, F. S., Martins, L. G. N., Oliveira, P. E. S., Teichrieb, C., Silva, S. M., Maroneze, R., Bodmann, B., Mortarini, L., Cava, D., Anfossi, D. (2018). Monitoring the micrometeorology of a coastal site next to a thermal power plant from the surface to $140 \mathrm{~m}$. Bulletin of the American Meteorological Society, online first(0), 1-8. 
Campos, J. G., Acevedo, O. C., Tota, J., Manzi, A. O. (2009). On the temporal scale of the turbulent exchange of carbon dioxide and energy above a tropical rain forest in amazonia. Journal of Geophysical Research: Atmospheres, 114(D8).

Degrazia, G., Anfossi, D., Carvalho, J., Mangia, C., Tirabassi, T., Velho, H. C. (2000). Turbulence parameterisation for pbl dispersion models in all stability conditions. Atmospheric environment, 34(21), 3575-3583.

Haugen, D., Kaimal, J., Bradley, E. (1971). An experimental study of reynolds stress and heat flux in the atmospheric surface layer Quarterly Journal of the Royal Meteorological Society, 97(412), 168-180.

Højstrup, J. (1981). A simple model for the adjustment of velocity spectra in unstable conditions downstream of an abrupt change in roughness and heat flux. Boundary-Layer Meteorology, 21(3), 341-356.

Howell, J., Mahrt, L. (1997). Multiresolution flux decomposition. Boundary-Layer Meteorology, 83(1), $117-137$.

Kaimal, J. C., Wyngaard, J., Izumi, Y., Coté, O. (1972). Spectral characteristics of surface-layer turbulence. Quarterly Journal of the Royal Meteorological Society, 98(417), 563-589.

Mallat, S. G. (1989). A theory for multiresolution signal decomposition: the wavelet representation. IEEE transactions on pattern analysis and machine intelligence, 11(7), 674-693.

Panofsky, H. A., Dutton, J. A. (1984). Atmospheric turbulence. John Wyley and Sons, New York.

Vickers, D., Mahrt, L. (2006). A solution for flux contamination by mesoscale motions with very weak turbulence. Boundary-layer meteorology, 118(3), 431-447.

Otávio Costa Acevedo

Claudio Alberto Teichrieb

Franciano Scremin Puhales

Luis Gustavo Nogueira Martins

Gervásio Annes Degrazia

Samuel Martins Silva

Rafael Maroneze
Universidade Federal de Santa Maria, Santa Maria, RS, Brasil

E-mail: otavio@ufsm.br

Universidade Federal de Santa Maria, Santa Maria, RS, Brasil E-mail: pablo.deoliveira@gmail.com

Universidade Federal de Santa Maria, Santa Maria, RS, Brasil E-mail: teichrieb@gmail.com

Universidade Federal de Santa Maria, Santa Maria, RS, Brasil E-mail: franciano.puhales@ufsm.br

Universidade Federal de Santa Maria, Santa Maria, RS, Brasil E-mail: lgsm@gmail.com

Universidade Federal de Santa Maria, Santa Maria, RS, Brasil E-mail: gervasio.degrazia@gmail.com

Universidade Federal de Santa Maria, Santa Maria, RS, Brasil E-mail: samueltec@gmail.com

Universidade Federal de Santa Maria, Santa Maria, RS, Brasil E-mail: rafaelmaroneze@gmail.com 